\title{
Meta-Analysis of the Efficacy and Safety of Mirabegron Add-On Therapy to Solifenacin for Overactive Bladder
}

\author{
Yankai $\mathrm{Xu}^{1,2,}$, Ruihua $\mathrm{Liu}^{3, *}$, Chu Liu', Yuanshan $\mathrm{Cui}^{4}$, Zhenli Gao ${ }^{4}$ \\ ${ }^{1}$ Shandong University School of Medicine, Jinan, China \\ ${ }^{2}$ Department of Urology, Yantai Affiliated Hospital of Binzhou Medicine University, Yantai, China. \\ ${ }^{3}$ Department of Ultrasound, Yantai Yuhuangding Hospital Affiliated to Medical College of Qingdao University, Yantai, China \\ ${ }^{4}$ Department of Urology, Yantai Yuhuangding Hospital Affiliated to Medical College of Qingdao University, Yantai, China
}

Purpose: We performed a meta-analysis to evaluate the efficacy and safety of mirabegron add-on therapy to solifenacin for patients with overactive bladder $(\mathrm{OAB})$.

Methods: We conducted a systematic literature review to identify all randomized, double-blind, controlled trials (RCTs) of this combination (mirabegron and solifenacin) for OAB. Embase, MEDLINE, and the Cochrane Central Register of Controlled Trials were searched. A manual search was also performed to investigate relevant references from the retrieved studies. Results: Four publications describing 5 RCTs that compared combination therapy with solifenacin, including a total of 3,309 patients, were analyzed. The mean number of micturitions per 24 hours (mean difference [MD], $-0.45 ; 95 \%$ confidence interval [CI], -0.65 to $-0.26 ; \mathrm{P}<0.00001$ ), number of episodes of incontinence per 24 hours (MD, $-0.71 ; 95 \% \mathrm{CI},-0.14$ to -0.02 ; $\mathrm{P}=0.04$ ), volume voided per micturition, and number of urgency episodes per 24 hours demonstrated that combination therapy was more effective than solifenacin therapy alone. Safety assessments, including common treatment-emergent adverse events (odds ratio, $1.09 ; 95 \% \mathrm{CI}, 0.95-1.27 ; \mathrm{P}=0.23)$ and discontinuations due to adverse events $(\mathrm{P}=0.30)$, demonstrated that the combination therapy was well tolerated.

Conclusions: This meta-analysis suggests that mirabegron therapy as an add-on to solifenacin provides a satisfactory therapeutic effect for $\mathrm{OAB}$ symptoms with a low occurrence of side effects.

Keywords: Mirabegron; Solifenacin; Urinary Bladder, Overactive; Meta-analysis; Randomized Controlled Trials

- Conflict of Interest: No potential conflict of interest relevant to this article was reported.

\section{- HIGHLIGHTS}

- We performed a meta-analysis to evaluate the efficacy and safety of mirabegron add-on therapy to solifenacin for patients with OAB.

- This meta-analysis suggests that mirabegron therapy as an add-on to solifenacin provides a satisfactory therapeutic effect for OAB symp-

toms with a low occurrence of side effects.

\section{INTRODUCTION}

Overactive bladder $(\mathrm{OAB})$ is characterized by symptoms of urinary urgency, frequency, and nocturia with or without urgency incontinence in the absence of a lower urinary tract infection
[1]. OAB is a common multifactorial health disorder that is associated with detrimental effects on quality of life and a considerable economic burden [2]. The prevalence of $\mathrm{OAB}$ is high, with $12 \%-16 \%$ of adults in Europe, the United States, and Asia affected by this symptomatic syndrome [3].
Corresponding author: Zhenli Gao (iD https://orcid.org/0000-0002-2241-4242 Department of Urology, Yantai Yuhuangding Hospital Affiliated to Medical College of Qingdao University, NO.20 East Yuhuangding Road, Yantai 264000, China E-mail: 978946700@qq.com / Tel: +86-10-6691999 / Fax: +86-10-0535-6691999 *Yankai Xu and Ruihua Liu contributed equally to this study as co-first authors. Submitted: June 6, 2017 / Accepted after revision: August 3, 2017
This is an Open Access article distributed under the terms of the Cre(c)
commons.org/licenses/by-nc/4.0/) which permits unrestricted non-commercial use, distribution, and reproduction in any medium, provided the original work is properly cited. 
Antimuscarinic agents are the mainstay of oral pharmacotherapy for $\mathrm{OAB}$, but long-term treatment is limited by their insufficient efficacy and antimuscarinic agent-associated adverse events (AEs). In clinical practice, antimuscarinics are often initially prescribed; however, increasing the dose may exacerbate antimuscarinic AEs, such as constipation and dry mouth, and result in treatment discontinuation [4]. The efficacy of $\beta 3$ adrenergics, which are associated with a new safety profile that differs from that of antimuscarinics, has been shown [5]. Mirabegron is a selective $\beta 3$-adrenoceptor (AR) agonist [6] that is the drug of first choice for OAB. Given that $\beta 3$-adrenergics and anticholinergics have distinct mechanisms of action, the combination of both drugs is a possible option and has been tested in several clinical trials $[7,8]$.

We performed a meta-analysis to evaluate the safety and efficacy of the combination of solifenacin (5 or $10 \mathrm{mg}$ ) plus mirabegron (50 mg) compared with solifenacin (5 or $10 \mathrm{mg}$ ) monotherapy (the most widely used doses in clinical practice) for OAB treatment.

\section{MATERIALS AND METHODS}

\section{Search Strategy}

A systematic search of the literature was undertaken using Embase (1993 to May 2017), MEDLINE (1966 to May 2017), and the Cochrane Central Register of Controlled Trials to retrieve published randomized controlled trials (RCTs) investigating the use of mirabegron add-on therapy to solifenacin for OAB. We searched the database using combinations of the following terms: "mirabegron, solifenacin, OAB, and RCT." Additionally, the references of the retrieved articles were screened.

\section{Inclusion Criteria}

The following criteria were used to select the RCTs: (1) mirabegron add-on therapy to solifenacin was analyzed in the study, (2) the full text of the selected study was accessible, and (3) accurate data were provided and could be analyzed (including the values of each parameter that was analyzed and the total number of subjects). When the same group of researchers investigated a certain subject group in multiple experiments, each study was included. Additionally, the most recently published study was included in the meta-analysis if an identical study was published in distinct journals or at a different time point. The flowchart in Fig. 1 depicts the selection process.

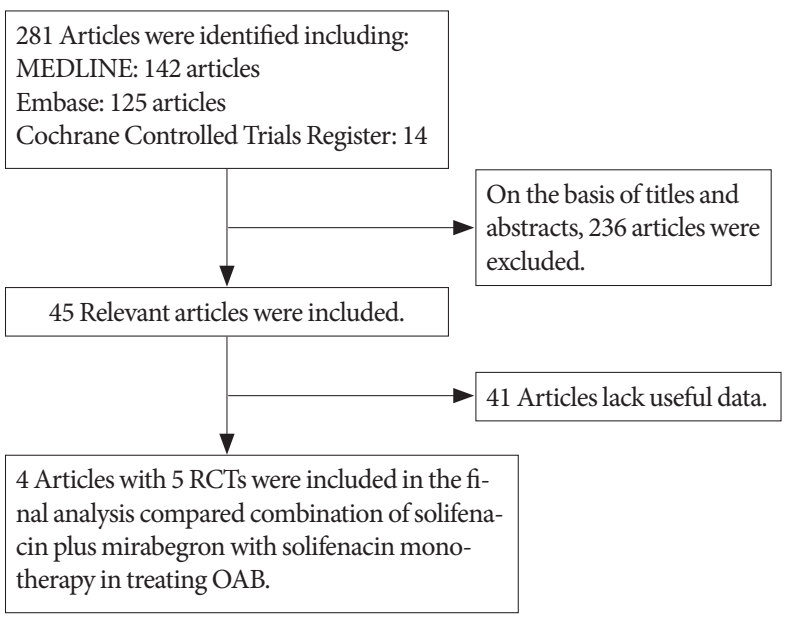

Fig. 1. Flow diagram of the study selection process. RCT, randomized controlled trial; OAB, overactive bladder.

\section{Quality Assessment}

The Jadad scale was used to determine the quality of the retrieved RCTs [9]. The methodological quality of each study was assessed according to the patient allocation method, blinding method, concealment of allocation procedures, and data loss due to discontinuation. Then, the studies were qualitatively classified according to the guidelines presented in the Cochrane Handbook for Systematic Reviews of Interventions version 5.1 .0 [10]. Each study was assigned and rated according to the following quality categories: A, the study had a low risk of bias if all quality criteria were adequately fulfilled; $B$, the study had a moderate risk of bias if one or more quality criteria were merely partially met or were ambiguous; or $\mathrm{C}$, the study had a high risk of bias if one or more of the criteria were barely met or were not included. Differences were settled by discussion between the authors.

\section{Data Extraction}

The following usable information was extracted from each study: (1) first author's name; (2) country of the study; (3) sample size; (4) year of publication; (5) therapy for the patients; and (6) data on the mean number of micturitions per 24 hours, mean number of episodes of incontinence per 24 hours, mean volume voided per micturition, mean number of urgency episodes per 24 hours, common treatment-emergent adverse events (TEAEs), and discontinuations due to AEs.

\section{Statistical Analysis and Meta-Analysis}

The meta-analysis was conducted using RevMan version 5.1.0. (Cochrane Collaboration, Oxford, UK) [10]. Differences be- 
tween the entry and endpoint of the study were evaluated according to the changes in the mean number of micturitions per 24 hours, mean number of episodes of incontinence per 24 hours, mean volume voided per micturition, mean number of urgency episodes per 24 hours, discontinuations due to AEs, and common TEAEs. Fixed-effects and random-effects models were used to assess the mean difference (MD) for continuous data and the odds ratio (OR) for dichotomous results pooled across studies with the corresponding 95\% confidence interval [CI] [11]. A study was considered homogeneous only when the analysis showed a P-value of $>0.05$, in which case a fixed-effects model was chosen for the meta-analysis; otherwise, a random-effects model was considered more appropriate.

\section{RESULTS}

\section{Characteristics of the Individual Studies}

The database search yielded 281 articles. A total of 236 studies were removed after reviewing the titles and abstracts of the articles according to the inclusion and exclusion criteria described above. A total of 41 of the remaining 45 articles were excluded due to a lack of useful data, and as a result, 4 articles describing 5 RCTs [12-16] that compared the combination of solifenacin (5 or $10 \mathrm{mg}$ ) plus mirabegron (50 mg) to solifenacin (5 or $10 \mathrm{mg}$ ) monotherapy were included in the analysis (Fig. 1). Table 1 presents relevant information about the articles.

\section{Quality of the Individual Studies}

All 5 RCTs were double-blinded, and all the articles elaborated on their randomization processes. All the included RCTs conducted a power calculation to determine the optimal sample size (Table 2). The level of quality of each study was A (Table 2). The funnel plot generated to provide a qualitative estimation of the publication bias of the studies suggested no evidence of bias (Fig. 2).

\section{Efficacy}

\section{Mean number of micturitions per 24 hours}

Five RCTs included data on the mean number of micturitions per 24 hours, for a cohort of 3,309 participants $(1,872$ in the combination group and 1,437 in the solifenacin group) (Fig. 3). The fixed-effects estimate of the MD was -0.45 , and the $95 \% \mathrm{CI}$ was -0.65 to $-0.26(\mathrm{P}<0.00001)$. This result suggests that mirabegron add-on therapy to solifenacin showed superior efficacy in reducing the mean number of micturitions per 24 hours com-

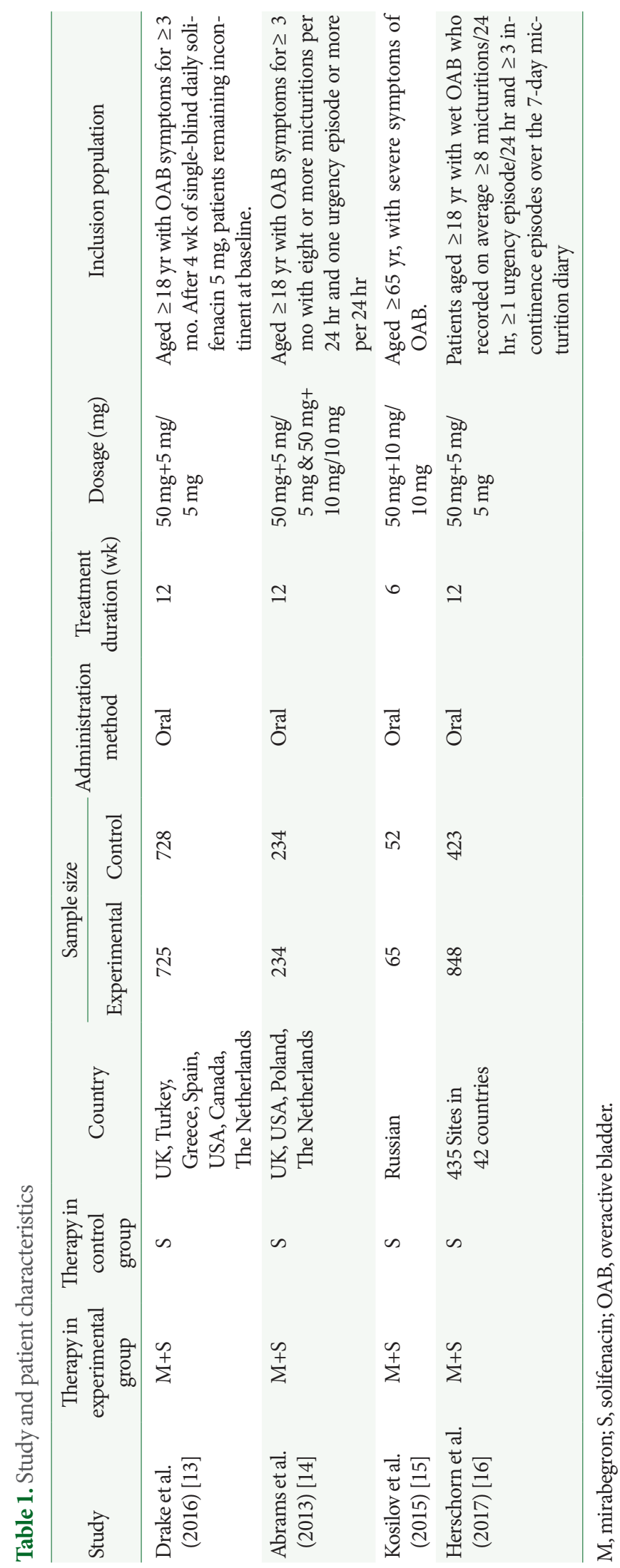


Table 2. Quality assessment of individual study

\begin{tabular}{cccccccc}
\hline Study & $\begin{array}{c}\text { Allocation } \\
\text { sequence } \\
\text { generation }\end{array}$ & $\begin{array}{c}\text { Allocation } \\
\text { concealment }\end{array}$ & Blinding & $\begin{array}{c}\text { Loss to } \\
\text { follow-up }\end{array}$ & $\begin{array}{c}\text { Calculation of } \\
\text { sample size }\end{array}$ & $\begin{array}{c}\text { Statistical } \\
\text { analysis }\end{array}$ & ITT analysis \\
Level of quality
\end{tabular}

A, all quality criteria met (adequate): low risk of bias; ITT, intention-to-treat; ANCOVA, analysis of covariance.

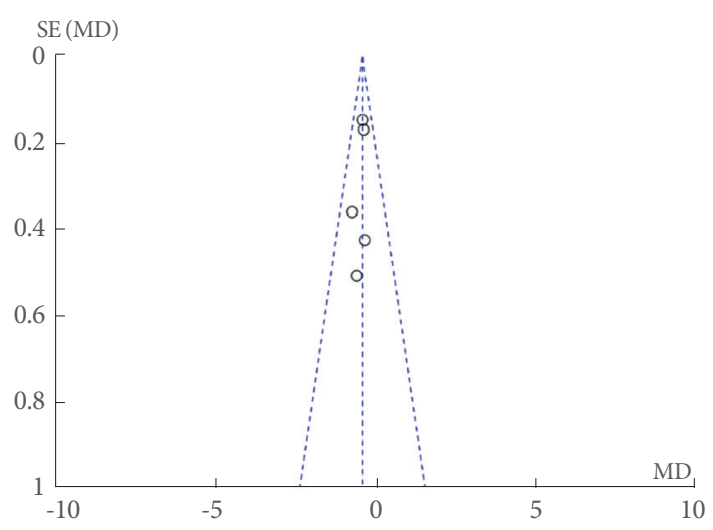

Fig. 2. Funnel plot of the studies included in our meta-analysis. $\mathrm{MD}$, mean difference; SE, standard error.

pared with solifenacin alone.

\section{Mean number of episodes of incontinence per 24 hours}

Five RCTs included data on the episodes of incontinence per 24 hours for a total of 3,309 participants (1,872 in the combination group and 1,437 in the solifenacin group) (Fig. 3). The randomeffects estimate of the MD was -0.71 , and the $95 \%$ CI was -1.40 to $-0.02(\mathrm{P}=0.04)$. This result indicates that the combination group experienced greater decreases in episodes of incontinence per 24 hours.

\section{Mean volume voided per micturition}

Four RCTs with a total of 3,192 participants (1,807 in the combination group and 1,385 in the solifenacin group) (Fig. 4) were identified. The fixed-effects estimate of the MD was 11.94, and the $95 \%$ CI was 8.02 to $15.87(\mathrm{P}<0.00001)$. This result suggests that the combination group experienced greater increases in the mean volume voided per micturition.

\section{Mean number of urgency episodes per 24 hours}

Three RCTs with a total of 1,921 participants (959 in the combination of solifenacin plus mirabegron group and 962 in the solifenacin group) (Fig. 4) were identified. The fixed-effects estimate of the MD was -0.56 , and the $95 \%$ CI was -0.83 to -0.30 $(\mathrm{P}<0.0001)$. This result suggests that mirabegron add-on therapy to solifenacin successfully reduced the mean number of urgency episodes per 24 hours.

\section{Safety}

\section{Treatment-emergent adverse events}

Five RCTs with a total of 3,309 participants (1,872 in the combination group and 1,437 in the solifenacin group) included TEAE data (Fig. 5). The OR was 1.09, and the 95\% CI was 0.95 to $1.27(\mathrm{P}=0.23)$. This result indicates that the groups were similar in terms of the incidence of TEAEs.

\section{Discontinuation due to $A E s$}

Five RCTs with a total of 3,309 participants (1,872 in the combination group and 1,437 in the solifenacin group) reported discontinuation due to AEs (Fig. 5). The pooled estimate of the OR was 1.33 , and the $95 \% \mathrm{CI}$ was 0.78 to $2.27(\mathrm{P}=0.30)$. No apparent differences were found between the combination group and the solifenacin-only group in terms of discontinuation due to AEs.

\section{Urinary retention, dry mouth, and blurred vision}

Three RCTs, representing 2,841 participants (1,638 in the combination group and 1,203 in the solifenacin group), included data on urinary retention. The pooled estimate of the OR was 1.74 , and the $95 \% \mathrm{CI}$ was 0.55 to $5.45(\mathrm{P}=0.34)$. Five RCTs included data on dry mouth (OR, 0.99; 95\% CI, 0.73-1.33; P= 


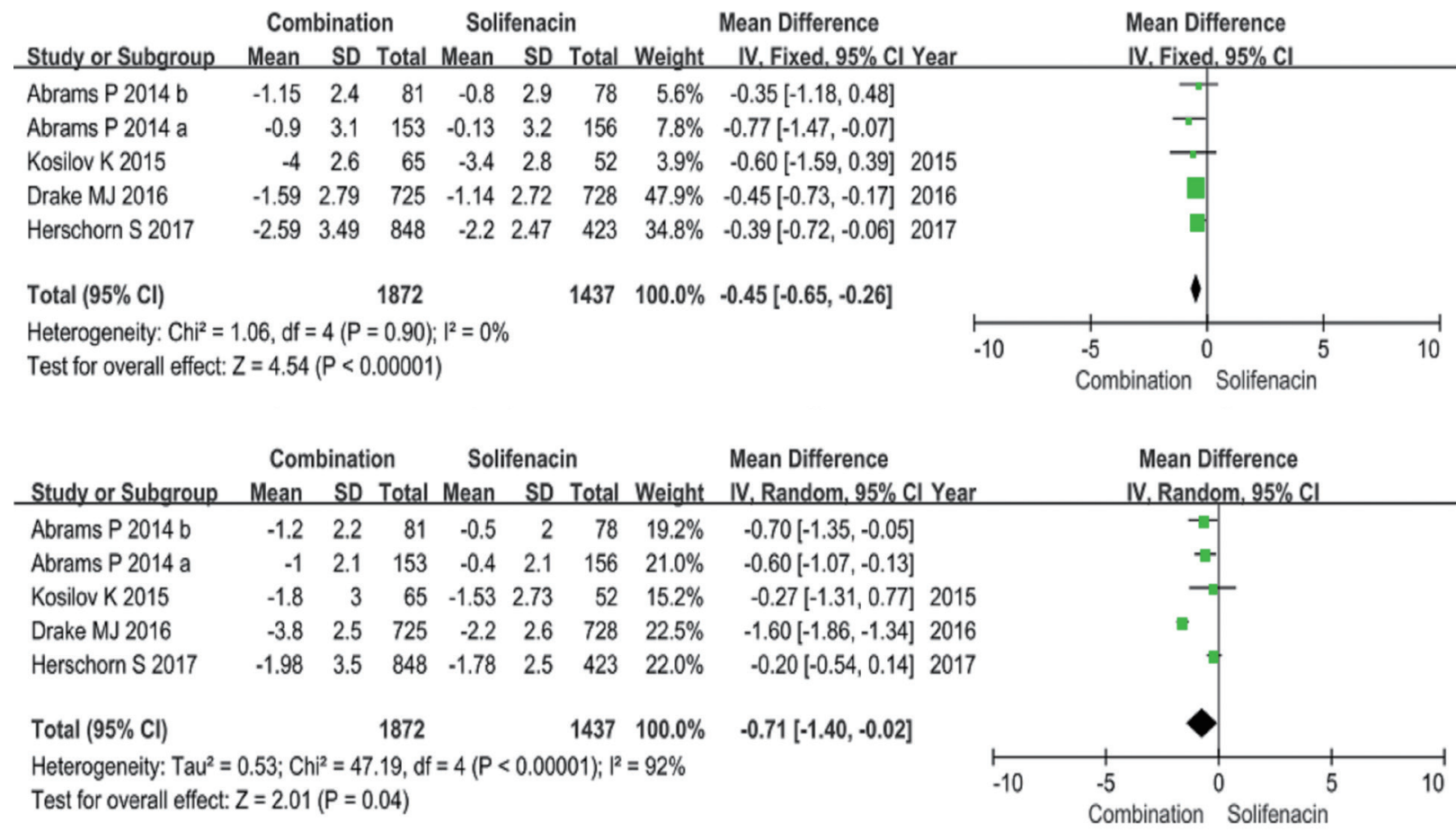

Fig. 3. Forest plots showing changes in the mean number of micturitions per 24 hours (A) and the number of episodes of incontinence per 24 hours (B). SD, standard deviation; IV, inverse variance; CI, confidence interval; df, degrees of freedom.

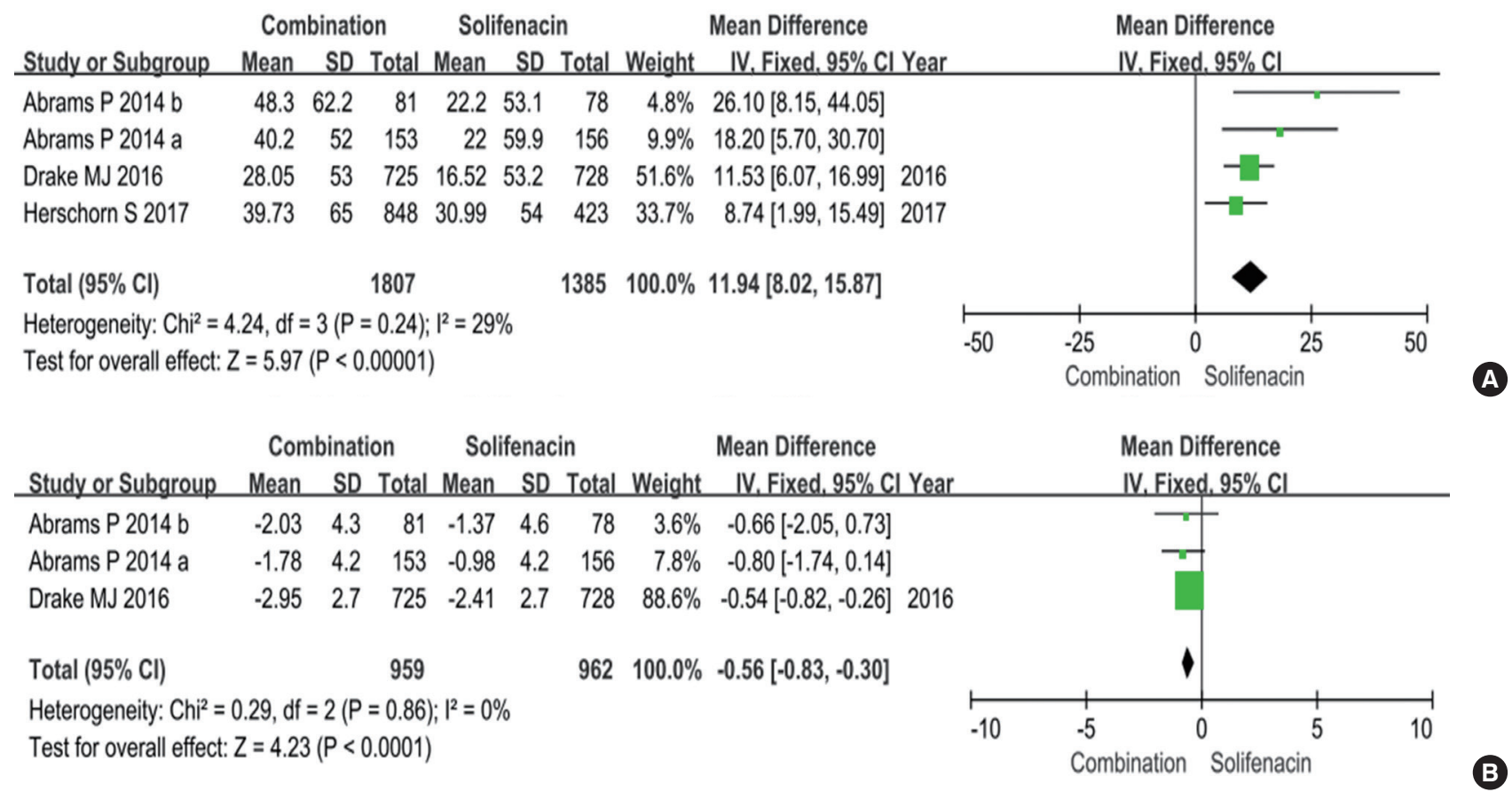

Fig. 4. Forest plots showing changes in the mean volume voided per micturition $(\mathrm{A})$ and the mean number of urgency episodes per 24 hours (B). SD, standard deviation; IV, inverse variance; CI, confidence interval; df, degrees of freedom. 


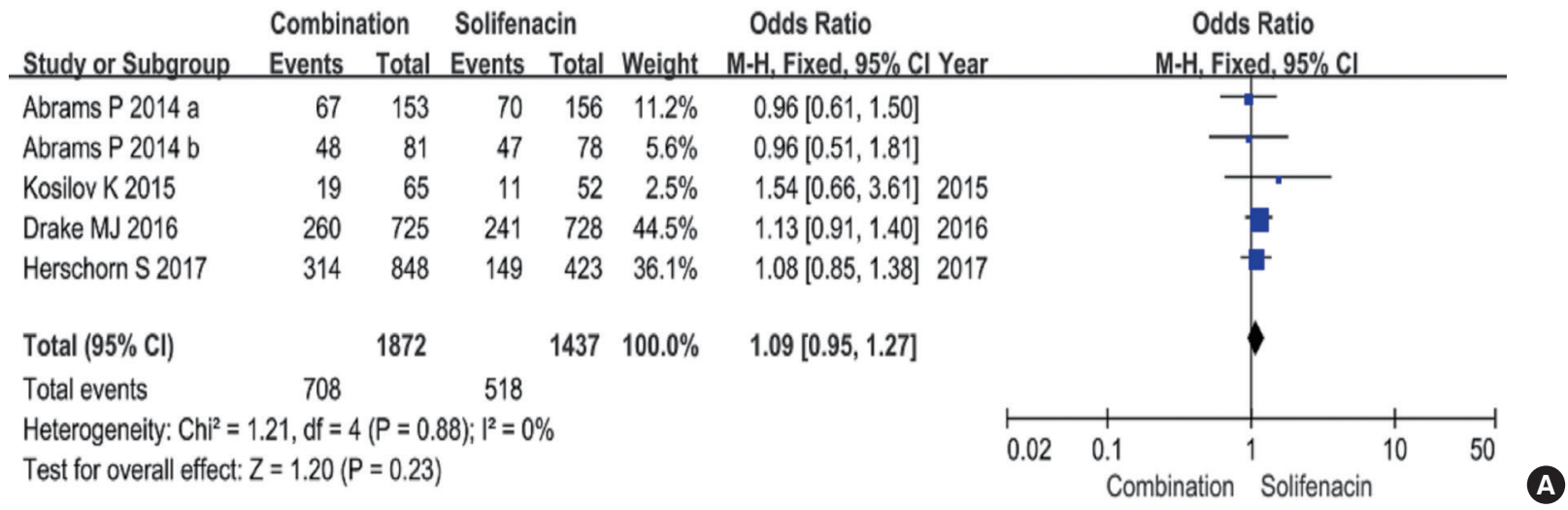

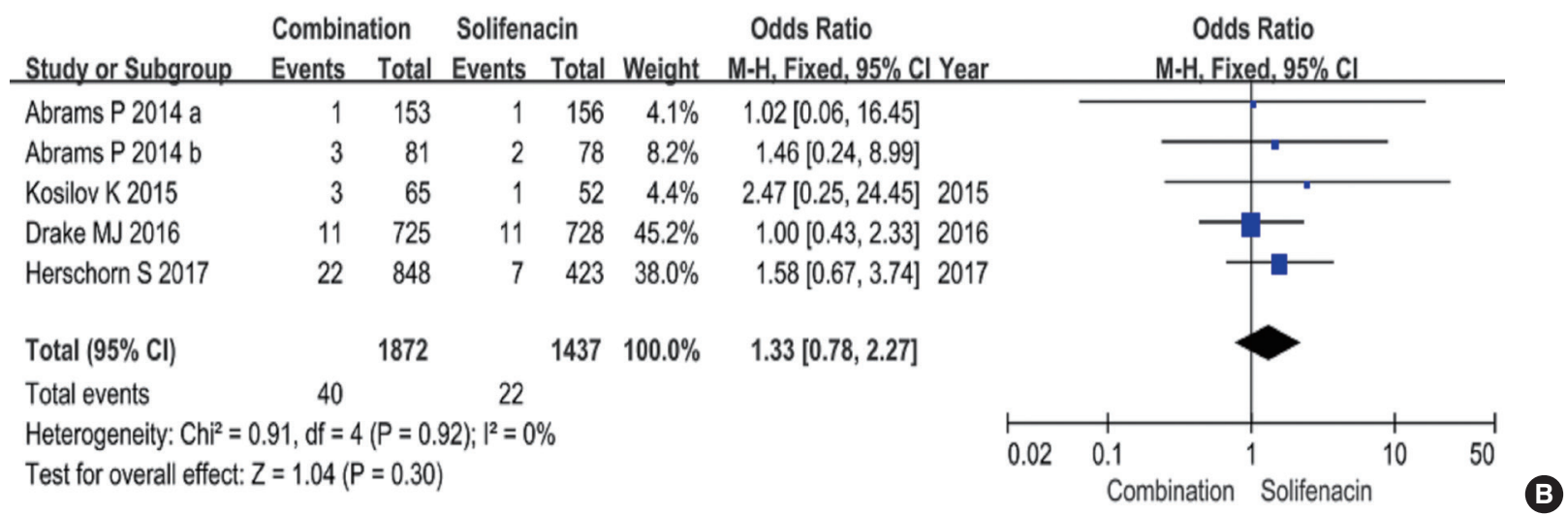

Fig. 5. Forest plots showing changes in treatment-emergent adverse events (A) and discontinuation due to adverse events (B). M-H, Mantel-Haenszel; CI, confidence interval; df, degrees of freedom.

$0.93)$, and 4 RCTs included data on blurred vision (OR, 1.80; 95\% CI, 0.52-6.18; $\mathrm{P}=0.35$ ). These results suggest that there were no apparent differences between the combination group and the solifenacin-only group in terms of the incidence of urinary retention, dry mouth, and blurred vision.

\section{DISCUSSION}

$\mathrm{OAB}$ is one of the most frequent lower urinary tract symptoms (LUTS) in both genders and is associated with significant bothersome symptoms and a poorer quality of life [17]. In many cases, no underlying cause is found, and the case of OAB is considered idiopathic [18]. Pharmacological therapy is widely used for $\mathrm{OAB}$ treatment. The 2 main classes of drugs used are antimuscarinics and the newer $\beta 3$-AR agonists [19]. Combining 2 oral pharmacotherapies with distinct modes of action and proven efficacy may improve $\mathrm{OAB}$ symptoms without exacerbating the burden of anticholinergic or $\beta 3$-AR agonists, which can obviate the need for dose escalation or more invasive interventions [20].
This systematic review and quantitative meta-analysis summarized the evidence from RCTs regarding the efficacy and safety of mirabegron (50 $\mathrm{mg}$ per day) add-on therapy to solifenacin (5 or $10 \mathrm{mg}$ per day) for OAB treatment. Our study demonstrated that the combination therapy was superior to solifenacin alone (5 or $10 \mathrm{mg}$ per day) in improving the mean number of micturitions per 24 hours, the mean number of episodes of incontinence per 24 hours, the mean volume voided per micturition, and the mean number of urgency episodes per 24 hours. Two RCTs $[13,14]$ evaluating the mean number of nocturia episodes, mean number of pads per 24 hours, and the $\mathrm{Pa}$ tient Perception of Bladder Condition score showed that adding mirabegron therapy to solifenacin was superior to solifenacin alone for improving OAB symptoms. One RCT [15] demonstrated that the postvoid residual bladder capacity, detrusor compliance, and maximum bladder capacity values significantly changed in the combination group. Overall, the combination therapy resulted in more pronounced improvements in multiple parameters. The findings indicate that combination therapy 
may be more useful than monotherapy for patients seeking additional symptomatic improvement.

Yamaguchi et al. [21] reported the results of the MILAI study, which was an open-label phase IV trial that demonstrated the effects of mirabegron as an add-on therapy in patients in whom $\mathrm{OAB}$ was treated with solifenacin. The authors discovered that the addition of mirabegron to solifenacin resulted in only mild to moderate AEs and had promising results in terms of efficacy. Imamura et al. [22] found that the different mechanisms of action of mirabegron and solifenacin were synergistic in inhibiting cold stress-induced detrusor overactivity in spontaneously hypertensive rats. Therefore, the combination of mirabegron and solifenacin offered significant improvements over single-drug treatment and lacked serious side effects in patients with cold stress-exacerbated LUTS.

When urine is stored, the binding of acetylcholine in the bladder activates afferent nerves to the muscarinic receptors (MRs) [23]. MR antagonists can effectively suppress the activation of the afferent nerves by blocking MRs. Additionally, MR antagonists may desensitize the bladder afferent nerves [24]. In contrast, $\beta 3$-AR agonists stimulate the release of nitric monoxide (NO) by the urothelium [25] by binding to receptors within the urothelium. NO suppresses the activation of the afferent nerves, and $\beta 3$-AR activation has an inhibitory effect on bladder afferent activity. Moreover, the combination of antimuscarinic and $\beta 3$ AR agonist agents was found to inhibit part of the Rho kinase pathway in vitro [26]. As a result, the combination of mirabegron and solifenacin may effectively improve bladder function during the storage phase by inhibiting afferent nerve activation.

The safety data included in this meta-analysis suggest that the combination of mirabegron and solifenacin is well tolerated. Measures of adverse reactions, such as discontinuations due to AEs and common TEAEs, were similar in the combination group and the solifenacin-only group. This result demonstrates the safety of mirabegron add-on therapy to solifenacin for $\mathrm{OAB}$ treatment. Dry mouth, which was the most frequently reported $\mathrm{AE}$ with antimuscarinics [27] and a common reason for treatment discontinuation [4], did not show a different pattern of incidence in the combination versus solifenacin-only groups. The vital signs, electrocardiogram parameters, and postvoid residual volume in the combination group showed no synergistic effects beyond those known from either monotherapy. Therefore, mirabegron (50 $\mathrm{mg}$ per day) add-on therapy to solifenacin (5 or $10 \mathrm{mg}$ per day) seems to offer a good balance between side effects and efficacy.
This meta-analysis included only double-blind RCTs. The quality of the individual studies in the meta-analysis was high based on the quality assessment scale that we developed. Nevertheless, few studies were included. The long-term safety, efficacy, and persistence of combination therapy cannot be extrapolated from this article, and the present analysis excluded data from unpublished studies. These factors may contribute to bias. Although the combination of either $5 \mathrm{mg}$ or $10 \mathrm{mg}$ of solifenacin with $50 \mathrm{mg}$ of mirabegron was found to be effective, the impacts of add-on treatment over monotherapy according to solifenacin dosage ( $5 \mathrm{mg}$ vs. $10 \mathrm{mg}$ ) might be different. In this regard, it would be preferable to show that there was no difference in the efficacy and safety according to the solifenacin dosage. However, such a comparison could not be conducted because of small number of RCTs that were included. More high-quality trials with larger sample sizes should further investigate the efficacy and safety of mirabegron add-on therapy to solifenacin for $\mathrm{OAB}$. Furthermore, cost is an important factor, particularly for older adults who may have high out-of-pocket costs associated with these drugs.

This meta-analysis indicates that mirabegron add-on therapy to solifenacin provides a satisfactory therapeutic effect for $\mathrm{OAB}$ symptoms without increasing the risk of side effects.

\section{REFERENCES}

1. Abrams P, Cardozo L, Fall M, Griffiths D, Rosier P, Ulmsten U, et al. The standardisation of terminology of lower urinary tract function: report from the Standardisation Subcommittee of the International Continence Society. Neurourol Urodyn 2002;21:167-78.

2. Sacco E, Tienforti D, D’Addessi A, Pinto F, Racioppi M, Totaro A, et al. Social, economic, and health utility considerations in the treatment of overactive bladder. Open Access J Urol 2010;2:11-24.

3. Homma Y, Yamaguchi O, Hayashi K; Neurogenic Bladder Society Committee. An epidemiological survey of overactive bladder symptoms in Japan. BJU Int 2005;96:1314-8.

4. Benner JS, Nichol MB, Rovner ES, Jumadilova Z, Alvir J, Hussein $\mathrm{M}$, et al. Patient-reported reasons for discontinuing overactive bladder medication. BJU Int 2010;105:1276-82.

5. Cui Y, Zong H, Yang C, Yan H, Zhang Y. The efficacy and safety of mirabegron in treating $\mathrm{OAB}$ : a systematic review and meta-analysis of phase III trials. Int Urol Nephrol 2014;46:275-84.

6. Takasu T, Ukai M, Sato S, Matsui T, Nagase I, Maruyama T, et al. Effect of (R)-2-(2-aminothiazol-4-yl)-4'-\{2-[(2-hydroxy-2-phenylethyl)amino]ethyl\} acetanilide (YM178), a novel selective beta3- 
adrenoceptor agonist, on bladder function. J Pharmacol Exp Ther 2007;321:642-7.

7. Serati M, Leone Roberti Maggiore U, Sorice P, Cantaluppi S, Finazzi Agrò E, Ghezzi F, et al. Is mirabegron equally as effective when used as first- or second-line therapy in women with overactive bladder? Int Urogynecol J 2017;28:1033-9.

8. Nomura Y, litsuka H, Toyoshima J, Kuroishi K, Hatta T, Kaibara A, et al. Pharmacokinetic drug interaction study between overactive bladder drugs mirabegron and tolterodine in Japanese healthy postmenopausal females. Drug Metab Pharmacokinet 2016;31: 411-6.

9. Jadad AR. Randomised controlled trials. London: BMJ Publishing Group; 1998.

10. Higgins JP, Green S, editors. Cochrane handbook for systematic reviews of interventions. version 5.1.0 [Internet]. The Cochrane Collaboration, 2011 [uptated 2011 Mar; cited 2017 May 10]. Available from: www.cochrane-handbook.org.

11. DerSimonian R, Laird N. Meta-analysis in clinical trials. Control Clin Trials 1986;7:177-88.

12. Higgins JP, Thompson SG, Deeks JJ, Altman DG. Measuring inconsistency in meta-analyses. BMJ 2003;327:557-60.

13. Drake MJ, Chapple C, Esen AA, Athanasiou S, Cambronero J, Mitcheson D, et al. Efficacy and safety of mirabegron add-on therapy to solifenacin in incontinent overactive bladder patients with an inadequate response to initial 4-week solifenacin monotherapy: a randomised double-blind multicentre phase 3B study (BESIDE). Eur Urol 2016;70:136-45.

14. Abrams P, Kelleher C, Staskin D, Rechberger T, Kay R, Martina R, et al. Combination treatment with mirabegron and solifenacin in patients with overactive bladder: efficacy and safety results from a randomised, double-blind, dose-ranging, phase 2 study (Symphony). Eur Urol 2015;67:577-88.

15. Kosilov K, Loparev S, Ivanovskaya M, Kosilova L. A randomized, controlled trial of effectiveness and safety of management of $\mathrm{OAB}$ symptoms in elderly men and women with standard-dosed combination of solifenacin and mirabegron. Arch Gerontol Geriatr 2015;61:212-6.

16. Herschorn S, Chapple CR, Abrams P, Arlandis S, Mitcheson D, Lee KS, et al. Efficacy and safety of combinations of mirabegron and solifenacin compared with monotherapy and placebo in patients with overactive bladder (SYNERGY study). BJU Int 2017 Apr 18 [Epub]. https://doi.org/10.1111/bju.13882.

17. Agarwal A, Eryuzlu LN, Cartwright R, Thorlund K, Tammela TL, Guyatt GH, et al. What is the most bothersome lower urinary tract symptom? Individual- and population-level perspectives for both men and women. Eur Urol 2014;65:1211-7.

18. Cornu JN. Combination of solifenacin and mirabegron for overactive bladder management. BJU Int 2015;116:498-9.

19. Griebling TL. Re: A randomized, controlled trial of effectiveness and safety of management of $\mathrm{OAB}$ symptoms in elderly men and women with standard-dosed combination of solifenacin and mirabegron. J Urol 2016;195:1834.

20. Abrams P, Kelleher C, Staskin D, Kay R, Martan A, Mincik I, et al. Combination treatment with mirabegron and solifenacin in patients with overactive bladder: exploratory responder analyses of efficacy and evaluation of patient-reported outcomes from a randomized, double-blind, factorial, dose-ranging, Phase II study (SYMPHONY). World J Urol 2017;35:827-38.

21. Yamaguchi O, Kakizaki H, Homma Y, Igawa Y, Takeda M, Nishizawa O, et al. Safety and efficacy of mirabegron as 'add-on' therapy in patients with overactive bladder treated with solifenacin: a postmarketing, open-label study in Japan (MILAI study). BJU Int 2015; 116:612-22.

22. Imamura T, Ogawa T, Minagawa T, Nagai T, Suzuki T, Saito T, et al. Combined treatment with a $\beta 3$-adrenergic receptor agonist and a muscarinic receptor antagonist inhibits detrusor overactivity induced by cold stress in spontaneously hypertensive rats. Neurourol Urodyn 2017;36:1026-33.

23. Lips KS, Wunsch J, Zarghooni S, Bschleipfer T, Schukowski K, Weidner W, et al. Acetylcholine and molecular components of its synthesis and release machinery in the urothelium. Eur Urol 2007; 51:1042-53.

24. Iijima K, De Wachter S, Wyndaele JJ. Effects of the M3 receptor selective muscarinic antagonist darifenacin on bladder afferent activity of the rat pelvic nerve. Eur Urol 2007;52:842-7.

25. Otsuka A, Shinbo H, Matsumoto R, Kurita Y, Ozono S. Expression and functional role of beta-adrenoceptors in the human urinary bladder urothelium. Naunyn Schmiedebergs Arch Pharmacol 2008;377:473-81.

26. Frazier EP, Peters SL, Braverman AS, Ruggieri MR Sr, Michel MC. Signal transduction underlying the control of urinary bladder smooth muscle tone by muscarinic receptors and beta-adrenoceptors. Naunyn Schmiedebergs Arch Pharmacol 2008;377:449-62.

27. Chapple CR, Khullar V, Gabriel Z, Muston D, Bitoun CE, Weinstein $\mathrm{D}$. The effects of antimuscarinic treatments in overactive bladder: an update of a systematic review and meta-analysis. Eur Urol 2008;54:543-62. 